\title{
Locating the 'missing wedge' artifacts from limited-angle CT reconstruction
}

\author{
Jianhong Liu, Yong Guan*, Liang Chen, Haobo Bai, Wenbin Wei, Yangchao Tian and Gang Liu.
}

National Synchrotron Radiation Laboratory, University of Science and Technology of China, Hefei, Anhui, People's Republic of China.

*Yong Guan, yongg@ustc.edu.cn

\section{Abstract:}

'Missing wedge' problem exists in some kind of CT imaging situations, such as electron microscopy, $\mathrm{x}-$ ray nano-CT image, etc. Method such as iterative reconstruction algorithms, total variation based method were applied to improve the reconstruction quality, but the 'missing wedge' artifacts are still inevitable. In this paper, a method based on image processing technique was proposed to locate the 'missing wedge' artifacts in CT reconstruction. The result showed good performance on locating the artifacts, which also showed the potential in CT reconstruction and image analysis in nano-CT.

Introduction:

In electron microscopy (EM) and X-ray nano-CT, 'missing wedge' problem is difficult to solve [1]. For the biological cell imaging experiment in the EM or the soft X-ray microscopy, mesh grids are used to carry the biological specimen. When imaging in the high tilt, the grid wall will shadow on the specimen cell, which makes the electron beam or the soft X-ray beam unable to transmit the specimen cell. Therefore, only limited-angle range projections can be acquired and the image reconstructions are incomplete and distorted. This is when the 'missing wedge' problem occurs, and it will cause some inconvenience in the further segmentation and image analysis. Few methods were proposed to reduce the 'missing wedge' problem, such as iterative reconstruction algorithms and total Variation (TV) based method[2], which do relatively reduce the 'missing wedge' problem. Moreover, the Discrete Algebraic Reconstruction Technique (DART) [1,3-7] was reported to achieve a high quality reconstruction with the limited-angle range projections, by using the prior knowledge of the composition of a limited number of materials and the boundaries between materials are sparse. In this paper, a method based on image segmentation and mathematical morphology operations was proposed to locate the majority of the 'missing wedge' artifacts in CT image reconstruction, which may help improve the reconstruction quality of the DART reconstruction algorithm. Simulation result showed the precise location of artifacts.

\section{Method:}

First, an input reconstruction image was needed for the following procedure. In this paper, the input reconstruction image was computed by the simultaneous algebraic reconstruction technique (SART). After the input reconstruction image was acquired, segmentation process was applied on it. In this paper, over-segmentation method based on thresholding method was used because the 'missing wedge' artifacts in the input reconstruction image would be segmented into many small areas, which would help to locate them in the next procedure. The detail over-segmentation procedure are as followed:

$$
\begin{gathered}
y(x)=\left\{\max (h(i)) \mid x-w_{1} \leq i \leq x+w_{1}\right\} \\
y_{1}(x)=\left\{\begin{array}{cc}
y(x), & \text { if } y^{\prime}(i)=0, x-w_{2} \leq i \leq x+w_{2} \\
0, & \text { else }
\end{array}\right. \\
\left\{x_{p} \mid y_{1}\left(x_{p}\right)>c_{t h r}, y_{1}\left(x_{p}\right)=h\left(x_{p}\right)\right\}
\end{gathered}
$$




$$
x_{v, i}=\underset{x \in\left(x_{p, i}, x_{p, i+1}\right)}{\arg \min }(h(x))
$$

The $h(x)$ is the smoothed histogram of the image. Equation (1-3) represent the peak-picking process. $w_{1}$ and $w_{2}$ represent the peak resolution. $C_{t h r}$ is a threshold for filtering the noise peaks. $x_{p}$ and $x_{v}$ are the point set of the peaks' and valleys' location, respectively. Then a gradient process was applied on the segmentation image, and a boundary image was acquired. Mathematical morphology operations were then apply on the boundary image. Figure 2.B shows the erosion operator and the dilation operator. First the erosion operation was applied on the boundary image by the erosion operator. Then the dilation operation was applied on the eroded boundary image by the dilation operator.

Result:

The phantom image in Figure 1.A was used to test the method. 141 projections in the angle range of 0 $140^{\circ}$ with $1^{\circ}$ angular increment were acquired. The input reconstruction image computed by the SART method in 500-iteration are shown in Figure 1.B. Segmentation image was showed in Figure 1.C. Then the boundary image was showed in Figure 2.A after the gradient process. After the erosion and dilation operation by the operator in Figure 2.B, the process image was showed in Figure 2.C. The absolute value of the difference between Figure 1.A and 1.B, which means the actual artifacts image, was shown
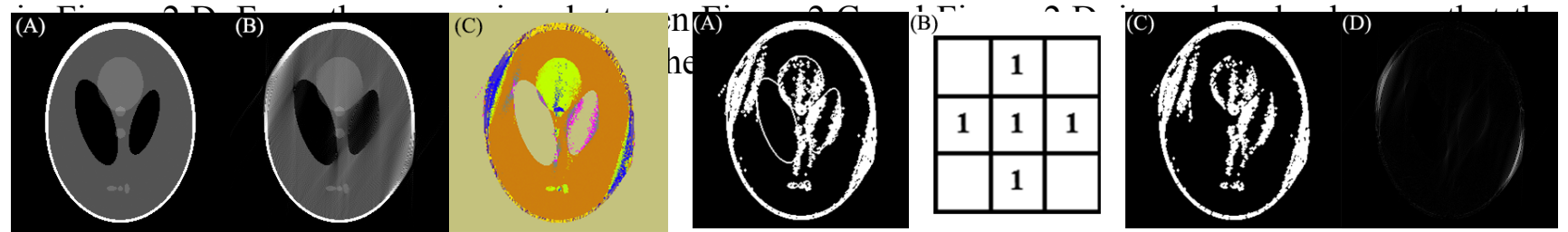

Figure. 1. (A) test phantom image; (B) input Figure. 2. (A) boundary image; (B) erosion and dilation image computed by SART; (C) segmentation operator; (C) location of the artifacts; (D) actual artifacts image.

Conclusion:

The method proposed in this paper showed good result in locating the 'missing wedge' artifacts. It shows the great potential in CT reconstruction and image analysis in the angle-limited reconstruction. It provides a new direction in $\mathrm{CT}$ reconstruction because the DART method also needs the segmentation and boundary-update process. Moreover, it also provides an additional option for the image analysis after the CT reconstruction for the EM or the soft X-ray nano-CT.

References:

[1] X Zhuge et al, Ultramicroscopy 175 (2017) 87-96.

[2] Z Liang et al, Proceedings of SPIE (2013) 8851.

[3] K Batenburg et al, IEEE International Conference on Image Processing ICIP (2007) 1829-1832.

[4] K Batenburg et al, Ieee Transactions on Image Processing 20 (2011) 2542-2553.

[5] W van Aarle et al, Ieee Transactions on Image Processing 21 (2012) 4608-4621.

[6] X Zhuge et al, Ieee Transactions on Image Processing 25 (2016) 455-468.

[7] Z Liang et al, Journal of Synchrotron Radiation 23 (2016) 606-616. 